\title{
Assigning Output Variables to Equations Using Linear Programming
}

PREM K. GUPTA and ARTHUR W. WESTERBERG

Department of Chemical Engineering

University of Florida, Gainesville, Florida 32611

JOHN E. HENDRY and RICHARD R. HUGHES

Department of Chemical Engineering

University of Wisconsin, Madison, Wisconsin 53706

Several papers have occurred in the literature on the subject of assigning output variables to equations in the course of deriving a solution procedure for the equations. Such an assignment is called an output set assignment.

Steward (1962) showed that if an output set assignment fails to exist, the equations are singular, that is, redundant or inconsistent. Given an output set assignment, effective algorithms exist to precedence order the equations (Harary, 1960; Steward, 1962; Sargent and Westerberg, 1964) and to locate minimum tear orderings within the irreducible groups found in the precedence order (Barkley and Motard, 1972). For nonsquare equation sets (that is, $n$ equations in $m$ variables, $m>n$ ), algorithms exist to find the output assignment which minimizes the number of tear variables resulting (Lee, Christensen, and Rudd, 1966) or to avoid singular equation sets being left for subsequent solution (Edie and Westerberg, 1971).

The subject has directly or indirectly therefore received considerable attention. The purpose of this note is to point out that the assigning of an output set for several criteria can be formulated as an Assignment Problem or a Bottleneck Problem in linear programming and thus solved by existing, very powerful algorithms for those problems.

\section{OUTPUT SET ASSIGNMENT PROBLEM}

First we shall define an index set for each equation $f_{i}$ :

$$
J(i)=\left\{j \mid x_{j} \text { occurs in } f_{i}\right\}
$$

Then an output set assignment may be defined as follows: For each equation $f_{i}, i=1,2, \ldots n$, assign a variable $x_{j}$, $j \in J(i)$. The assignment must be such that each $x_{j}, j=$ $1,2, \ldots m(\supseteq n)$, is assigned to at most one equation. We can represent the $k$ th assignment $O S A(k)$ by the ordered sequence of numbers

$$
\operatorname{OSA}(k)=\{j(k, 1), j(k, 2), \ldots, j(k, n)\}
$$

where $j(k, l)$ is the index of the variable assigned to equation $l$ in output assignment $k$.

\section{WEIGHTED OUTPUT ASSIGNMENT, LP1}

To each variable $x_{j}$ in equation $f_{i}$, assign a weight $W_{i j} \geqslant$ 0 . The weight should reflect the relative desirability of assigning the variable $x_{j}$ to $f_{i}$. Do this for every equation.

For convenience define

$$
W_{\max }=\operatorname{Max}\left\{W_{i j} \mid i=1,2, \ldots n ; j \in J(i)\right\}
$$

Then for each equation $i$ and variable $x_{j}, j \neq J(i)$, assign a negative weight of large magnitude such as $-n\left(W_{\max }+\right.$ 1) (or develop the Assignment Algorithm to ignore any

P. K. Gupta is with the Department of Chemical Engineering, University of Michigan, Ann Arbar, Michigan 48104. J. E. Hendry is with Conzine Riotinto of Australia, Ltd., Melbourne, Victoria 3001, Australia. variables $x_{j}, j \notin J(i)$, in $\left.f_{i}\right)$.

The weighted output assignment considered here then results from finding the output assignment $k$ which maximizes $\sum_{i=1}^{n} W_{i j(k, i)}$ where here $j(k, i)$ is again the index of the variable assigned to $f_{i}$ in assignment $k$. This problem is equivalent to solving the following linear programming problem which we shall call LP1:

so that

$$
\operatorname{Max}_{\epsilon_{i j}} \sum_{i=1}^{n} \sum_{j=1}^{m} W_{i j} \epsilon_{i j}
$$

all

$$
\begin{aligned}
& \sum_{j=1}^{m} \epsilon_{i j}=1, \quad i=1,2, \ldots n \\
& \sum_{i=1}^{n} \epsilon_{i j} \leq 1, \quad i=1,2, \ldots m
\end{aligned}
$$

$$
\epsilon_{i j} \equiv 0 \text {, }
$$

The constraints effectively constrain each $\epsilon_{i j}$ to lie in the range $0 \leq \epsilon_{i j} \leq 1$. An optimum solution to this problem will be at an extreme point, and each extreme point has the property that each $\epsilon_{i j}$ is either 0 or 1 . If $\epsilon_{i j}$ is either 0 or 1 , then constraints (1) and (2) combined force us to find exactly one $\epsilon_{i j}=1$ in each equation $f_{i}$ and no more than one $\epsilon_{i j}=1$ in each column $j$.

Thus if $\epsilon_{p q}=1$ at the solution, we assign variable $q$ to equation $p$. Note, if the objective $\underset{i}{\sum} \sum_{j} W_{i j} \epsilon_{i j}<0$ at the solution to LP1, then no output assignment exists for the equation set and the equations are singular. LP1 is the form of an Assignment Problem in linear programming.

\section{WEIGHTED OUTPUT ASSIGNMENT, LP2}

Another useful form for a weighted output assignment can also be proposed (Hendry, 1972). Here we assign a weight $W_{i j}>0$ for $j \epsilon J(i)$ and $W_{i j}=0$ for $j \notin J(i)$. Again the weight reflects the desirability of assigning the variable $x_{j}$ to $f_{i}$. The objective changes, however; we wish in this case to choose an output assignment which maximizes the minimum desirability $W_{i j}$ associated with any of the assigned variables. The associated linear programming problem, which we shall call LP2, has the form

$\operatorname{Max}_{\epsilon_{i j}, \gamma} \gamma$

$$
\begin{aligned}
W_{i j} \epsilon_{i j} \geqslant \gamma \quad & i=1,2, \ldots, n \\
\text { and } \quad j & =1,2, \ldots, m
\end{aligned}
$$

plus constraints (1), (2), and (3) of problem LP1. Again, because of constraints (1), (2), and (3), we obtain an 
output set assignment if one exists. If $\gamma$ at the solution is zero, no output assignment exists for the equation set. LP2 is the form of a Bottleneck Problem in linear programming. Again a very efficient solution algorithm exists (Garfinkel, 1971).

\section{DIFFERENT CRITERION FOR CHOOSING THE $W_{i j}$}

Assigning an arbitrary output set. Choose all $W_{i j}, j \in J(i)$, equal to one. Assign the remaining $W_{i j}$ as before. Then the solution to LPI or LP2 will be an arbitrary assignment.

Maximum Product Assignment. Westerberg and Edie (1971b) developed the maximum product criterion for assigning an output set. The criterion was developed to find the output assignment with hopefully the best convergence properties if a solution procedure is derived based on it. The objective, for $n$ equations in $n$ unknowns, is to find the assignment which maximizes the product

$$
\operatorname{Max}_{k}\left|\prod_{i} \frac{\partial f_{i}}{\partial x_{j(k, i)}}\right|_{x}
$$

at a guessed solution point $\hat{x}$. We define

and

$$
b_{i j}=\left|\frac{\partial f_{i}}{\partial x_{j}}\right|_{\hat{x}}
$$

$$
b_{\min }=\operatorname{Min}_{i, j}\left\{b_{i j} \mid b_{i j} \neq 0\right\}
$$

Then the weights $W_{i j}$ in LP1 can be chosen as

$$
W_{i j}= \begin{cases}\log \left(b_{i j} / b_{\text {min }}\right) & b_{i j} \neq 0 \\ \text { negative number of } & b_{i j}=0 \\ \text { large magnitude } & \end{cases}
$$

Note, LP2 could be used but the implications have not been investigated. Also note that, for LP2, we set all $W_{i j}$ $=0$ for the case $b_{i j}=0$ above.

Kevorkian and Snoeck Criterion. In a paper by Kevorkian and Snoeck (1973), a criterion was stated for choosing the output set based on relative errors. Unfortunately the presentation is not complete, but it appears their criterion is the same as the maximum product criterion. This results because the maximum product criterion is independent of row and column scaling, and thus rescaling each column in the Jacobian to reflect relative errors rather than absolute errors will not change the resulting assignment. Thus it appears their problem can also be converted to a linear programming problem of the form of LPI.

Minimize Maximum Row Sum Norm. Westerberg and Edie (1971a) proposed several criteria based on local iteration operator norms for choosing output sets for a system of $n$ equations in $n$ unknowns. To choose the output set, the equations are linearized in terms of perturbation variables about an assumed solution. The criteria for choosing an output assignment were then associated with minimizing one of three possible norms for the matrix operator associated with a Jacobi iteration scheme (Carnahan et al., 1969). Hendry (1972) reformulated one of the criteria, the row sum norm criterion, as a Bottleneck Problem. Using the nomenclature of the previous section, the contribution to the row sum norm of the local Jacobi iteration operator for assigning variable $x_{j}$ to equation $f_{i}$ is

By choosing

$$
r_{i j}=\left[\sum_{\substack{k=1 \\ k \neq j}}^{n} b_{i k}\right] / b_{i j}, b_{i j} \neq 0
$$

$$
W_{i j}=\left\{\begin{array}{lll}
\text { A large number, } & & r_{i j}=0 \\
1 / r_{i j} & , \quad b_{i j} \neq 0, & r_{i j} \neq 0 \\
0 & , & \text { Otherwise }
\end{array}\right.
$$

the minimum row sum norm criterion is satisfied by solving as the problem LP2. [Hendry (1972) demonstrated the effectiveness of choosing an output assignment using this criterion by deriving a solution procedure based on it for a fairly simple multistage reactor system described by Frank (1967). Frank chose an apparently reasonable solution method and then had great difficulty in arriving at a numerical solution. The procedure found by Hendry converged readily.]

Algebraic Simplicity. Lee and Ozawa (1971) suggested assigning weights to reflect algebraic properties of the equations and variables. For example, if an equation $f_{i}$ can be conveniently rearranged algebraically to solve explicitly for the variable $x_{j}$, then $W_{i j}$ can be set high whereas if the variable is only implicitly given by the equation, its weight can be set very low. An example might be in the equation which defines log mean temperature driving force.

$$
\Delta T_{l m}=\frac{\left(T_{1}-t_{1}\right)-\left(T_{2}-t_{2}\right)}{\ln \left(\frac{T_{1}-t_{1}}{T_{2}-t_{2}}\right)}
$$

Here only the variable $\Delta T_{l m}$ is conveniently found as an output variable.

\section{NONSQUARE SYSTEMS}

For nonsquare systems, $n$ equations in $m>n$ variables, the acyclic algorithm of Lee, Christensen, and Rudd (1966) should be used first in an attempt to find an assignment which leaves a set of equations and variables which fully precedence order. In a nonsquare system, the $m-n$ variables not assigned to equations become the decision variables for the problem, and, if some variables are preferred as decision variables they can be given low weights in all equations in which they occur to make them less attractive as output variables. If they must be decisions, they can obviously be treated as not being in the equations in the first place.

\section{DISCUSSION}

This paper is based on the independent work of two research groups: (1) Hendry and Hughes and (2) Gupta and Westerberg. The latter group was made aware of solving the maximum product criterion problem as a linear programming problem by independent inputs from McWhite and Thomas (1970) and Edie (1970). At the same time the former group discovered the same result (Hendry, 1972).

In a master's project report by Gupta (1972), an explicit statement of the Assignment Algorithm is given, and it has been implemented in the GENDER system (Cunningham and Westerberg, 1972). The algorithm uses the so-called "Hungarian Algorithm" for solving the linear programming problem. In this algorithm partial output assignments are readily extended. Thus one can add an equation to or delete an equation from a set already having an assignment and modify rather quickly the existing assignment to account for the modification. Similarly one can add or remove columns, that is, variables.

\section{ACKNOWLEDGMENT}

This work was supported in part by NSF Grant GK18633 (Florida) and also in part by the University of Wisconsin Graduate School and Wisconsin Alumni Research Foundation. 


\section{LITERATURE CITED}

Barkley, R. W., and R. L. Motard, "Decomposition of Nets," Chem. Eng. J., 3, 265 (1972).

Carnahan, B., H. A. Luther, and J. O. Wilkes, Applied Numerical Methods, 298-299, Wiley, New York (1969).

Cunningham, J. R., and A. W. Westerberg, "The GENDER Routines, An Aid for the Analysis and Optimization of Engineering Systems," Proc. of the 6th Annual Princeton Conf. on Info. Sci. and Systems, Princeton, N. J. 48-52 (1972).

Edie, F. C., private communication (1970).

Da, and A. W. Westerberg, "Computer Aided Design Part 3-Decision Variable Selection to Avoid Hidden Singularities in Resulting Recycle Calculations," Chem. Eng. J., 2, 114 (1971).

Frank, A., Chem. Eng. Pragr. Symp. Ser. No. 72, 63, 54 (1967).

Harary, F., "On the Consistency of Precedence Matrices," J. Assoc. Comp. Mach., 7, 255 (1960).

Hendry, J. E., "Computer Aided Synthesis of Optimal Multicomponent Separation Sequences," 197-218 PhD thesis, Univ. Wisconsin, Madison (1972).

Kevorkian, A. K., and J. Snoeck, "Decomposition in Large Scale Systems-Theory and Applications in Solving Large Sets of Non-linear Simultaneous Equations," in Decomposi- tion of Large-Scale Problems, D. M. Himmelblau (ed), American Elsevier, New York, 491-515 (1973).

Lee, W., J. H. Christensen, and D. F. Rudd, "Design Variable Selection to Simplify Process Calculations," AIChE J., 12, 1104 ( 1966 ).

Lee, W., and Y. Ozawa, "Complex Chemical Engineering Systems," Chem. Eng. Educ., 5, 144 (1971).

McWhite, P., and M. E. Thomas, private communication (1970).

Sargent, R. W. H., and A. W. Westerberg, "'SPEED-UP' in Chem. Engineering Design," Trans. Instn. Chem. Engrs., 42, T190 (1964).

Steward, D. V., "On an Approach to Techniques for the Analysis of the Structure of Large Systems of Equations," SIAM Review, 4, 321 (1962).

Westerberg, A. W., and F. C. Edie, "Computer Aided Design Part 1-Enhancing Convergence Properties by the Choice of Output Variable Assignment in the Solution of Sparse Equation Sets," Chem. Eng. J., 2, 9 ( 1971).

-., "Computer Aided Design Part 2-An Approach to Convergence and Tearing in the Solution of Sparse Equation Sets," ibid., 17.

Manuscript received October 30 and accepted December 27, 1973.

\title{
Prediction of the Dew Point Locus in Methane-Light Hydrocarbon Binary Systems in the Neighborhood of the Methane Critical Point
}

\author{
Y. ARAI, R. J. J. CHEN, \\ P. S. CHAPPELEAR, and R. KOBAYASHí
}

Department of Chemical Engineering Rice University, Houston, Texas 77001

Chen et al. (1972) have developed an elution technique for the determination of the dew point concentration of binary mixtures in the 98 to 100 mole \% range. The experimental results of Chen et al. (1973) exhibit a phenomena which could not be observed with less accurate methods. The dew point isotherm is multiple valued in the region $\left(\sim 5^{\circ} \mathrm{C}\right)$ about the critical temperature $T_{c}$ of the more volatile component. Isotherms at temperatures slightly above $T_{c}$ are quadruple valued, as observed in methane- $n$-pentane (Chen et al., 1973); and at $T_{c}$ and slightly lower temperatures the isotherms are triple valued or $S$ shaped, as observed in methane- $n$-butane (Chen et al., 1973). This phenomena has been termed double retrograde vaporization by Carnahan et al. (1972).

The present study evaluates the ability of the Benedict-
Webb-Rubin (BWR) (1951) equation of state for the computation of these phenomena.

\section{COMPUTATION}

The BWR equation was used in an iterative fashion to calculate fugacities, various derivatives, and $K$-values as required. The ordinary BWR combining rules with the modification proposed by Stotler and Benedict (1953) were used:

$$
A_{0}=y_{1}^{2} A_{01}+2 y_{1} y_{2} m \sqrt{A_{01} A_{02}}+y_{2}{ }^{2} A_{02}
$$

which introduces a binary interaction parameter $m$.

The points A, B, C, D on Figure 1 have certain mathematical restrictions which may be used for computational 